\title{
Diacronie
}

Studi di Storia Contemporanea

$N^{\circ} 28,4 \mid 2016$

La voce del silenzio

\section{Lilia Moritz Schwarcz, Heloisa Murgel Starling, Brasil: Uma Biografia}

Brasile: una biografia

\section{Andreza Santos Cruz Maynard}

Traduttore: Jacopo Bassi

\section{OpenEdition}

\section{Journals}

\section{Edizione digitale}

URL: http://journals.openedition.org/diacronie/4439

DOI: 10.4000/diacronie.4439

ISSN: 2038-0925

\section{Editore}

Association culturelle Diacronie

Notizia bibliografica digitale

Andreza Santos Cruz Maynard, «Lilia Moritz Schwarcz, Heloisa Murgel Starling, Brasil: Uma Biografia », Diacronie [Online], № 28, 4 | 2016, Messo online il 29 décembre 2016, consultato il 23 septembre 2020. URL : http://journals.openedition.org/diacronie/4439; DOI : https://doi.org/10.4000/diacronie.4439 


\section{Diacronie}

N. 28 | 4|2016 La voce del silenzio: intelligence, spionaggio e conflitto nel XX secolo

16/

\section{PANORAMICA: Brasile 2015}

Francisco das Chagas F. SANTIAGO Júnior, Daniel CHAVES, Petrônio DOMINGUES, Mairon Escorsi VALÉRIO, Andreza Santos Cruz MAYNARD, Dilton Cândido Santos MAYNARD, Karl SCHURSTER *

\section{SILVA, Francisco Carlos Teixeira, LEÃO, Karl Shurster Sousa, LAPSKY, Igor (org.), O Cinema Vai à Guerra [Il cinema va in guerra], Rio de Janeiro, Campus, 2015, 274 pp.}

a cura di Francisco das Chagas F. SANTIAGO Júnior

traduzione e adattamento di Jacopo BASSI

O Cinema vai à Guerra, libro curato da Francisco Carlos T. da Silva, Karl Schurster Leão e Igor Lapsky si inserisce nel solco della tradizione storiografica brasiliana attenta alla relazione fra storia e cinema. Il tema della guerra nel cinema non è nuovo né nello scenario internazionale, né in Brasile, non essendo, peraltro, monopolio della ricerca storica. La maggior parte delle monografie precedenti, infatti sono state scritte - in ambito brasiliano - da studiosi o critici cinematografici. O cinema vai à guerra è incentrato sulla guerra intesa come topos della storia del tempo presente e della rappresentazione del passato (lontano e prossimo). Nella ottica secondo cui la narrazione cinematografica sarebbe «la principale concorrente della narrazione storica»1, i testi della collettanea riconoscono il ruolo del cinema nell'elaborazione visiva della coscienza storica dei secoli XX e XXI e del suo funzionamento come produttore di immagini del presente e del passato che finiscono per comporre la memoria delle comunità nazionali. Alcune problematiche-concetti percorrono molti o quasi tutti i testi: tra queste spiccano quelle di identità, allegoria e conflitto/guerra. L'insieme dei testi di $\mathrm{O}$ cinema vai à guerra, come lo stesso titolo suggerisce, costituisce

\footnotetext{
${ }^{1}$ SILVA, Francisco Carlos Teixeira, LEÃO, Karl Shurster Sousa, LAPSKY Igor (org.), O Cinema Vai à Guerra, Rio de Janeiro, Campus, 2015, p. XI.
} 
un campo di dibattito storiografico sul modo in cui le pellicole prendono parte e trasformano le guerre in quotidiane esperienze storiche degli spettatori.

TEIXEIRA DA SILVA, Francisco Carlos, MEDEIROS, Sabrina, VIANNA, Alexander Martins (orgs.), Enciclopédia de Guerras e Revoluções do Século XX [Enciclopedia delle guerre e delle rivoluzioni nel $X X$ secolo], 3 voll., Rio de Janeiro, Campus/Elsevier, 2014-2015, 496 + 304 + 672 pp. a cura di Daniel Santiago CHAVES RIBEIRO traduzione e adattamento di Jacopo BASSI

L'Enciclopédia de Guerras e Revoluções do Século XX si colloca nel solco di una tradizione contraddistinta da opere che sono presto divenute di riferimento, a cui in Brasile hanno fatto ricorso differenti generazioni per avvicinarsi a questo campo di studi nell'ambito delle scienze umane. In questo caso la tradizione non si è consolidata sui dizionari ed enciclopedie, ma si può dire che quest'opera rinnovi la tradizione possedendo un carattere proprio, autonomo, plurale e autoriale.

Nella sinossi i curatori presentano le loro scelte autoriali affermando che «si tratta di opere complete che presentano idee, movimenti, fatti e personaggi che modellarono l'inizio del secolo tanto nel campo della politica e dell'economia, quanto delle arti e delle scienze».

Il ruolo di sforzi genuini come quello di questa Enciclopédia, nei suoi tre rinnovati volumi, è quello di espandere il carattere olistico di questo dibattito nel promuovere una raccolta potenzialmente euristica non solamente su temi di interesse storico, ma anche sul ruolo del pensatore contemporaneo inteso come costruttore di una comprensione totale, antiutilitarista e antiframmentaria, che legge i tempi con una lanterna portata a mano, inserito in un dibattito pubblico totalizzante $\mathrm{e}$ onnicomprensivo. In questo senso l'opera, densa e ampia, ci invita a un'intensa passeggiata nella totalità come una questione propria delle scienze umane, che non può essere spiegata da qualche sortita dilettantesca e isolata, ma solamente attraverso un incontro di idee. 
MACHADO, Maria Helena Pereira Toledo, CASTILHO, Celso Thomas (Orgs.), Tornando-se livre: agentes históricos e lutas sociais no processo de Abolição [Liberandosi: ragioni storiche e lotte sociali nel processo abolizionista], São Paulo, EDUSP, 2015, 480 pp.

a cura di Petrônio DOMINGUES

traduzione e adattamento di Jacopo BASSI

Come si realizzò il processo che portò alla fine della schiavitù in Brasile? Soprattutto a partire dal protagonismo degli schiavi, degli affrancati, ma anche, genericamente, dai brasiliani? Quali furono i meccanismi alla base della conquista della libertà e i limiti di questi nel contesto schiavista e in quello successivo all'abolizione della schiavitù? In altri termini, come si verificò il processo emancipazionista nella principale nazione dell'America Latina? E nel periodo successivo all'abolizione della schiavitù come poté realizzarsi uno scenario costellato dalle lotte per i diritti e la cittadinanza? Domande a cui non è facile dare risposta e intorno alle quali ruota Tornando-se livre: agentes históricos e lutas sociais no processo de Abolição.

Il libro è una raccolta collettanea articolata in 21 capitoli, scritti da diversi storici, provenienti da università brasiliane ed estere (europee e statunitensi).

Come la maggior parte delle collettanee, il risultato non è uniforme per quel che riguarda le riflessioni degli autori, la forma espositiva e lo stile e rivela in alcuni casi riflessioni originali, in altre occasioni riassunti (o note) di ricerche presentate per i corsi di laurea o critiche storiografiche, benché i testi siano sempre interessanti. Si nota la reiterazione di idee e di analisi da parte degli autori che restituisce l'impressione di un "vai e vieni"; questo emerge soprattutto nel complesso dei testi, ma alle volte anche all'interno di uno stesso testo. Questo non compromette in alcun modo la qualità della raccolta, che condensa alcune delle principali tendenze di studio riguardo alla schiavitù e al periodo successivo all'abolizione della schiavitù, un campo tematico emergente e promettente della storiografia brasiliana. 
RIBEIRO, Renilson Rosa, O Brasil inventado pelo Visconde de Porto Seguro: Francisco Adolfo de Varnhagen, o Instituto Histórico e Geográfico Brasileiro e a construção da ideia de Brasil-Colônia no BrasilImpério (1838-1860) [Il Brasile inventato dal Visconte di Porto Seguro: Francisco Adolfo de Varnhagen, l'Instituto Histórico e Geográfico Brasileiro e la construzione dell'idea di Brasile-colonia nel Brasile-impero (1838-186o)], Cuiabá, Entrelinhas, 2015, 448 pp.

a cura di Mairon Escorsi VALÉRIO

traduzione e adattamento di Jacopo BASSI

L'opera tratta delle tappe fondanti della storiografia nazionale - fissate intorno alla figura di Francisco Adolfo de Varnhagen e della sua opera História Geral do Brasil -, del ruolo esercitato in questo processo dall'IHGB (Instituto Histórico e Geográfico Brasileiro) e del ruolo centrale che lo sviluppo di una narrazione storica del Brasile coloniale esercitò nella creazione del Brasile monarchico del Secondo Impero.

Il libro è diviso in tre capitoli, ciascuno contenente una propria tesi, che concorrono nella parte conclusiva ad un solo esito: l'invenzione storica del Brasile Colonia è parte di un progetto politico di costruzione dello Stato nazionale improntata sul modello del Secondo regno.

Renilson R. Ribeiro si propone di «fare la storia dell'emergere di un oggetto, di un sapere, di un tempo e di uno spazio di potere: il passato coloniale brasiliano» ${ }^{2}$. In questa triade, il passato stabilisce il campo d'azione del sapere in questione, la storia; il termine coloniale marca il periodo definito e, infine, l'aggettivo brasiliano punta alla progettazione di un territorio. All'interno di questa triade, il popolo è il soggetto e le sue azioni rappresentano gli episodi attraverso cui costruire una storia marcata dalla linearità e dalla continuità fino al processo di consolidamento come Stato indipendente.

Dunque, O Brasil inventado pelo Visconde de Porto Seguro è un libro che pone nuovamente una questione importante per coloro che lavorano per la produzione della conoscenza: da chi o da cosa è prodotta la conoscenza? A chi serve il mestiere di storico? Che tipo di relazioni gerarchiche di autorità legittima? A che tipo di relazioni di potere è indissolubilmente legato?

Tra le diverse questioni affrontate da Renilson R. Ribeiro questa sembra essere fondamentale. Varnhagen era cosciente che il suo progetto di scrivere una storia del

\footnotetext{
2 RIBEIRO, Renilson Rosa, O Brasil inventado pelo Visconde de Porto Seguro: Francisco Adolfo de Varnhagen, o Instituto Histórico e Geográfico Brasileiro e a construção da ideia de Brasil-Colônia no Brasil-Império (1838-1860), Cuiabá, Entrelinhas, 2015, p. 46
} 
Brasile serviva una causa politica, ossia, aveva una chiara finalità: aiutare a impilare i mattoni dello Stato monarchico brasiliano fornendogli una storia dotata di costumi e di un’identità coerente.

SCHWARCZ, Lilia Moritz, STARLING, Heloisa Murgel, Brasil: Uma Biografia [Brasile: una biografia], São Paulo, Companhia das Letras, 2015, 792 pp.

a cura di Andreza Santos Cruz MAYNARD

traduzione e adattamento di Jacopo BASSI

Il libro è il risultato del lavoro congiunto di Lilia Moritz Schwarcz e Heloisa Murgel Starling: si tratta di una sintesi che affronta più di cinquecento anni di storia del Brasile.

L'analisi sviluppata nel libro parte dai primi contatti degli europei con gli indigeni brasiliani, all'inizio del XVI secolo, e giunge fino alla contemporaneità: per l'esattezza sino al periodo successivo al ritorno alla democrazia, con la ripresa del governo da parte dei civili, in seguito all'elezione di Fernando Henrique Cardoso alla presidenza, nel 1995.

Una delle particolarità che attirano maggiormente l'attenzione sul libro è la proposta delle autrici di partire dalla biografia per comprendere il Brasile in prospettiva storica.

Nell'introduzione vengono esposte alcune idee e metodi che pervadono l'opera. Da un lato apprezziamo l'uso dei riferimenti letterari, una delle caratteristiche che conferiscono leggerezza al testo. Al contempo, veniamo messi a parte delle nozioni di cittadinanza, democrazia, repubblica e giustizia sociale che verranno riprese nel corso dei capitoli. La visione critica delle autrici riguardo alla formazione storica del Brasile è permeata da queste categorie. L'altra particolarità del libro scritto da Schwarcz e Starling è la tendenza a ridurre al massimo i riferimenti ad autori ed opere, così come l'uso di citazioni e note. Queste sono rispettosamente collocate in fondo al volume in modo da non interrompere il flusso del testo.

Anche nell'introduzione, le autrici si avvalgono di riferimenti letterari come Lima Barreto e Guimaraes Rosa. Ma è di Gustave Flaubert - e del suo personaggio Madame Bovary - che si servono per rifarsi al bovarismo, richiamandosi così a Sérgio Buarque de Holanda; un concetto che fa riferimento ad un'alterazione nella percezione della realtà. Le autrici associano le affermazioni di Lima Barreto e Sérgio Buarque de 
Holanda per affermare che «i brasiliani hanno un qualcosa di Bovary»3. Secondo Schwarcz e Starling, il «bovarismo nazionale» si coagula con il "familismo", ossia l'abitudine di trasformare ciò che è pubblico in privato. È possibile comprendere come in questo risieda la ricerca dell'identità nazionale.

I decenni più recenti rimangono fuori dall'opera; le autrici giustificano questa scelta in considerazione del fatto che: «Il tempo presente appartiene un po' a ciascuno di noi e, probabilmente, tocca al giornalista prenderne nota con precisione e spirito critico» 4 . Marc Bloch discorderebbe dalle autrici su questo punto; nel libro A estranha derrota 5 , mostra quanto sia importante l'analisi che lo storico produce indagando il tempo presente.

Forse è questo il "tallone d'Achille" dell'opera: menzionare l'attualità senza imbarcarsi in un'analisi corrispondente. In altre parole rimarchiamo l'assenza di un'analisi della storia del tempo presente a fronte di riferimenti significativi ad esso. Questa insistenza nel menzionare fatti del presente senza l'accompagnamento di un'analisi critica risulta problematica sia dal punto di vista metodologico, sia da quello politico. Infine occorre puntualizzare che si tratta di un testo didascalico e analitico, ma soprattutto fluido e la cui lettura risulta piacevole. Si nota la preoccupazione delle autrici nel mantenere un linguaggio adatto ad un pubblico diversificato, non solamente accademico. L'opera risulta di immediato interesse per chi si occupa di storia del Brasile, ma può suscitare interesse anche al di fuori dell'ambito accademico.

\section{LOCHERY, Neill, Brasil: os frutos da guerra [Brasile: i frutti della} guerra], Rio de Janeiro, Intrínseca, 2015, 368 pp.

a cura di Dilton Cândido Santos MAYNARD

traduzione e adattamento di Jacopo BASSI

La Seconda guerra mondiale offrì al Brasile l'opportunità di divenire la nazione più influente dell'America del Sud, facendosi spazio come protagonista nei giochi di potere globali. Per questa ragione stipulare un alleanza con la Germania o gli Stati Uniti - le principali potenze prima dello scoppio della guerra - era qualcosa più che necessario. Era strategico. Al termine delle ostilità, benché si fosse scelto il lato dei vincitori, i risultati non furono quelli sperati.

3 SCHWARCZ, Lilia Moritz, STARLING, Heloisa Murgel, Brasil: Uma Biografia, São Paulo, Companhia das Letras, 2015, p. 16.

4 Ibidem, p. 20.

5 BLOCH, Marc, A estranha derrota, Rio de Janeiro, Jorge Zahar, 2011 [ed. orig. L'Étrange Défaite, Paris, Société des Éditions Franc-Tireur, 1946]. 
Quali furono, dunque, i risultati del coinvolgimento brasiliano nella Seconda guerra mondiale? Che cosa rimase al paese dello sforzo in cui si produsse? Quale fu il ruolo di Getúlio Vargas e del suo governo nei cambiamenti infrastrutturali, economici, nell'universo militare e nell'immagine del Brasile nel mondo? È questa la storia raccontata da Neill Lochery in Brasil: os frutos da Guerra.

Il libro è diviso in 6 parti, per un totale di 19 capitoli a cui si aggiungono le conclusioni. Un bell'album fotografico di 16 foto in bianco e nero arricchisce l'opera. Il repertorio di fonti utilizzate dall'autore è ampio e diversificato: diari, giornali, documenti, riviste, corrispondenza diplomatica (memorandum, telegrammi etc.), discorsi, e testimonianze raccolte negli archivi brasiliani, nordamericani e britannici.

L'opera di Lochery esplora il progetto nato con la cosiddetta "Politica di Buon Vicinato", concetto elaborato dal Segretario di Stato nordamericano Cordell Hull e dal diplomatico Sumner Welles e messo in opera da Franklin D. Roosevelt per garantire l'adesione dei paesi latinoamericani allo sforzo bellico statunitense.

L’idea di fondo del libro è quella per cui il Brasile moderno si sia costituito durante la Seconda guerra mondiale. Questa prospettiva appare già nel titolo originale Brazil: The fortunes of War. World War II and the making of modern Brazil.

Brasil: Frutos da Guerra è molto ben riuscita come opera generale. La passione dell'autore per il Brasile e per il periodo traspare da ogni pagina; la sua ammirazione per Vargas, Alzira e Oswaldo Aranha è innegabile. In definitiva questa storia dell'allora nascente Brasile moderno è sincera, il ché rappresenta la maggior virtù e il problema principale dell'opera.

TIBURI, Márcia, Como conversar com um fascista. Reflexões sobre o cotidiano autoritário brasileiro [Come parlare con un fascista. Riflessioni sulla quotidianità autoritaria brasiliana], Rio de Janeiro, Record, 2015, 194 pp.

a cura di Karl SCHURSTER

traduzione e adattamento di Jacopo BASSI

«Ciò che è in gioco è la riduzione dell'altro a un oggetto» ${ }^{6}$. Questa frase, tratta dall'ultimo libro della filosofa e artista gaúcha Márcia Angelita Tiburi forse è quella che meglio riassume tutto lo sforzo dell'autrice per far comprendere come agisca l'individuo fascista. Il suo intento principale è quello di analizzare, attraverso 67 brevi saggi, ciò

6 TIBURI, Márcia, Como conversar com um fascista. Reflexões sobre o cotidiano autoritário brasileiro, Rio de Janeiro, Record, 2015, p. 191. 
che rimane del fascismo nella società attuale e quanto sia capace di produrre una soffocante quotidianità autoritaria. Niente di più opportuno nell'attuale congiuntura politica brasiliana.

Il testo da cui nasce il libro, Como conversar com um fascista, è una piccola ma profonda riflessione su come diverse pratiche sociali, quando non instituzionali, abbiano prodotto nel Brasile contemporaneo l'estinzione della politica. Questo annichilimento di quella che sarebbe per eccellenza l'arte del dialogo, avrebbe generato il terreno fertile per la crescita dell'azione politica fascista e sarebbe presente nel nostro quotidiano attraverso ciò che la filosofia ha identificato come il «genocidio indigeno, il massacro razzista e classista contro giovani neri e poveri nelle periferie delle grandi città, la violenza domestica e l'assassinio delle donne, l'omofobia e la manipolazione dei bambini»7. La questione persistente risiede in questo caso nella capacità della società di creare meccanismi capaci di impedire la ripetizione di fenomeni di autoritarismo e di odio, attraverso una spiegazione rigorosa, adeguata e allo stesso tempo consistente, dei fenomeni dell'agire politico fascista in grado di negare l'esistenza dell'altro.

Attraverso l'interpretazione di Márcia Tiburi, è possibile capire che anche le democrazie consolidate dal punto di vista istituzionale, come quella brasiliana, corrono rischi costanti quando le pratiche fascistizzanti diventano una faccenda quotidiana.

7 TIBURI, Márcia, op. cit., p. 29. 


\section{* Gli autori}

Francisco das Chagas F. Santiago Júnior si è addottorato in storia presso l’Universidade Federal Fluminense, Niterói/Brasil con una tesi sull'appropriazione delle religioni afro-brasiliane nel cinema del periodo del regime dittatoriale degli anni Settanta. Lavora sulla relazione fra storia e cinema a partire da differenti assi di ricerca: il cinema e l'afro-brasilianità, la negoziazione del patrimonio culturale all'interno del cinema brasiliano, l'uso del passato nel cinema nazionale. Ha pubblicato numerosi articoli sulla cultura visuale, la teoria dell'immagine e la metodologia della ricerca multimediale.

URL: < http://www.studistorici.com/progett/autori/\#Santiago >

Daniel Santiago Chaves Ribeiro è professore associato di Storia contemporanea dell'Universidade Federal do Amapá (Unifap). Ha conseguito il Dottorato di ricerca in Storia comparata presso l'UFRJ (Universidade Federal do Rio de Janeiro), ed è un membro permanente del corpo docente del Corso di specializzazione Master in sviluppo regionale (PPGMDR); è ricercatore senior dell'Observatório de Fronteiras do Platô das Guianas (OBFRON) e del Círculo de Pesquisas do Tempo Presente (CPTP), entrambi afferenti all'Unifap.

URL: < http://www.studistorici.com/progett/autori/\#Chaves >

Petrônio Domingues si è addottorato in Storia presso l'USP (Universidade de São Paulo). Dal 2006 è professore dell'Universidade Federal de Sergipe e membro permanente del corpo docente del corso di Laurea specialistica in Storia (PROHIS) della stessa istituzione. Visiting Scholar della Rutgers - The State University of New Jersey (Stati Uniti),presso il Department of African Studies (2012-2013), è autore del libro, insieme a Flávio dos Santos Gomes, Da nitidez e invisibilidade: legados do pós-emancipação no Brasil (Belo Horizonte, Fino Traço Editora, 2013) e curatore - sempre assieme a Flávio Gomes - della raccolta Políticas da raça: experiências e legados da abolição e da pós-emancipação (São Paulo, Selo Negro Edições, 2014).

URL: < http://www.studistorici.com/progett/autori/\# Domingues >

Mairon Escorsi Valério si è addottorato in Storia culturale presso l'UNICAMP ed è professore associato dell'Universidade Federal da Fronteira Sul (UFFS) - Campus Erechim/RS. I suoi studi vertono sulla storia contemporanea dell'America Latina e sulla didattica della storia. Ł̀ autore del libro Entre a cruz e a foice: D. Pedro Casaldáliga e a significação religiosa do Araguaia (Jundiaí, Paco Editorial, 2012).

URL: < http://www.studistorici.com/progett/autori/\#Valerio > 
Andreza Santos Cruz Maynard si è laureata in Storia presso l'Universidade Federal de Sergipe, ha conseguito la Laurea specialsitica nell'Universidade Federal de Pernambuco e si è addottorata presso l'Universidade Estadual Paulista "Júlio de Mesquita Filho". Ricercatrice post-dottorale in Storia dell'Universidade Federal Rural de Pernambuco, è stata borsista CNPq/FAPITEC con una borsa DCR (2014-2016). Fa parte del Grupo de Estudos do Tempo Presente (GET/UFS/CNPq) ed è professoressa del Colégio de Aplicação dell'Universidade Federal de Sergipe.

URL: < http://www.studistorici.com/progett/autori/\#SantosCruzMaynard >

Dilton Cândido Santos Maynard si è laureato in Storia e ha conseguito la specializzazione in Sociologia presso l'Universidade Federal de Sergipe. Si è addottorato in Storia presso l'Universidade Federal de Pernambuco ed è stato ricercatore post-dottorale in Storia nell'Universidade Federal del Rio de Janeiro (UFRJ). Professore del Dipartimento di Storia, dei corsi di specializzazione in Scienze della formazione (PPGED/UFS) e del master professionalizzante in Storia (ProfHistória/UFS). Collabora in qualità di professore al corso di specializzazione in Storia comparata dell'UFRJ. Coordina il Grupo de Estudos do Tempo Presente (GET/UFS/CNPq).

URL: < http://www.studistorici.com/progett/autori/\#Maynard >

Karl Schurster è stato ricercatore post-doc e ha conseguito il dottorato in Storia presso l'UFRJ (Universidade Federal do Rio de Janeiro). Insegna come professore dell'Universidade de Pernambuco nel campo di studi della Storia del Tempo presente ed è membro permanente del corso di Laurea in Scienze della Formazione presso la stessa università. Attualmente sta realizzando un secondo stage postdottorale presso la Freie Universität Berlin sotto la direzione del professor Stefan Rinke. I suoi interessi sono rivolti allo studio della politica internazionale, con particolare interesse sui conflitti, in particolare le guerre mondiali e l'olocausto. Ha conseguito il $2^{\circ}$ posto al Premio Jabuti, assieme a Francisco Carlos Teixeira e Francisco Eduardo Almeida per aver curato: Atlântico: a história de um oceano, Rio de Janeiro, Editora Civilização Brasileira, 2013.

URL: < http://www.studistorici.com/progett/autori/\#Schurster > 


\section{Per citare questo articolo:}

SANTIAGO JÚNIOR, Francisco das Chagas F., CHAVES, Daniel Santiago Ribeiro, DOMINGUES, Petrônio, VALÉRIO, Mairon Escorsi, MAYNARD, Andreza Santos Cruz, MAYNARD, Dilton Cândido Santos, SCHURSTER, Karl, «Panoramica: Brasile 2015», Diacronie. Studi di Storia Contemporanea : La voce del silenzio: intelligence, spionaggio e conflitto nel XX secolo, 29/12/2016,

URL:<http://www.studistorici.com/2016/12/29/brasile_numero_28/ >

Diacronie Studi di Storia Contemporanea 3 www.diacronie.it

Risorsa digitale indipendente a carattere storiografico. Uscita trimestrale. redazione.diacronie@hotmail.it

Comitato di redazione: Jacopo Bassi - Luca Bufarale - Antonio César Moreno Cantano - Deborah Paci - Fausto Pietrancosta - Alessandro Salvador - Matteo Tomasoni - Luca Zuccolo

Diritti: gli articoli di Diacronie. Studi di Storia Contemporanea sono pubblicati sotto licenza Creative Commons 3.0. Possono essere riprodotti e modificati a patto di indicare eventuali modifiche dei contenuti, di riconoscere la paternità dell'opera e di condividerla allo stesso modo. La citazione di estratti è comunque sempre autorizzata, nei limiti previsti dalla legge. 